\title{
Hierarchical Control of a Low Voltage DC Microgrid with Coordinated Power Management Strategies
}

\author{
Shrishell Muchande \\ Department of Electrical Engineering \\ Fr. Conceicao Rodrigues Institute of Technology \\ Navi Mumbai, India \\ skmuchande@git-india.edu.in
}

\author{
Sushil Thale \\ Department of Electrical Engineering \\ Fr. Conceicao Rodrigues Institute of Technology \\ Navi Mumbai, India \\ sushil.thale@fcrit.ac.in
}

\begin{abstract}
A microgrid consists of a cluster of renewable energy sources, energy storage elements, and loads. One of the main objectives of a microgrid is to provide reliable and high-quality power to the loads. Under normal operating conditions, this is achieved through suitable Power Management Strategy (PMS). However, under emergency conditions, such as the failure of any source, overloads, or faults, the PMS may not be able to retain the microgrid in operating conditions. Any emergency condition may demand a significant change in control and coordination between various subsystems of the microgrid to survive and continue the operation. This feature makes a microgrid "a fault resilient" system as visualized in its objectives. This paper proposes a novel Coordinated Power Management (CPM) strategy based on three-layer hierarchical control for an autonomous Low Voltage DC (LVDC) microgrid. The proposed CPM strategy ensures the continuation of the microgrid operation under normal and emergency conditions. An emergency control layer is established to extend the microgrid operation during an emergency condition. The performance of the proposed control scheme is validated through simulation and experimental results.
\end{abstract}

Keywords-coordinated power management; DC microgrid; hierarchical control; fault resilient system

\section{INTRODUCTION}

Some of the challenges the conventional power generation faces are the environmental pollution, the reduction in the fossil fuels reservoirs, and the continuous increase in the power demand, which lead to increased penetration of the RES based Distributed Generation (DG). The resultant large penetration of RESs like Solar Photovoltaic (SPV) panels and wind turbines in existing grid is subsequently challenging its stability due to their intermittent nature. Small capacity sources with diverse nature integrated with Energy Storage Systems (ESSs) feeding aggregated loads are controlled and coordinated as a single entity, referred to as a Microgrid (MG). It facilitates the integration of free and locally available micro energy sources with suitable energy storage elements to meet the local load demand. In MGs, micro-sources are controlled through suitable power electronic converters. Based on voltage type, an MG can be classified as an AC MG, a DC MG or DCMG, and a Hybrid MG [1]. A DCMG possesses more advantages such as higher efficiency and natural interface with RES, ESS, and the loads.
Figure 1 shows a typical schematic of an LVDC MG configuration.

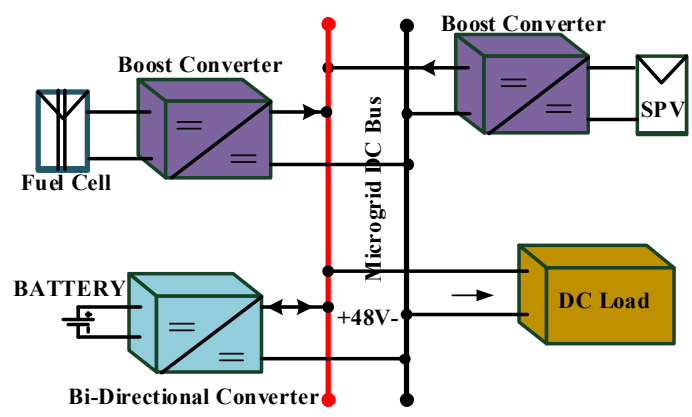

Fig. 1. A typical LVDC MG configuration.

The intermittent behavior of RES along with the unpredictable load fluctuations generally results in imbalance between the power generation and the load demand which significantly affects the overall operation of the system. Various power sharing control strategies have been presented in the past and are classified as decentralized, centralized and multilevel (hierarchical) control. In decentralized control, generally power management with droop control is adopted based on the capacity of individual sources. This method is simple and easy to implement with locally measured variables, but possesses some operational challenges like optimal Load Sharing (LS), voltage regulation, power regulation, real time source optimization etc. These challenges mainly persist due to the lack of information about source parameters. To address these issues, some improved droop control strategies have been proposed to enhance the load sharing accuracy, source optimization and voltage regulation achieved with low bandwidth communication $[2,3]$.

In centralized control, the sources are controlled by a remotely located centralized controller with high-speed communication links. The communication network with sufficiently large bandwidth plays an important role to exchange the information among the micro sources. However, the reliability of this centralized scheme suffers by the high probability of single point failures like the failures of the 
central controller or of the communication networks. The hierarchical control is a better approach which neither suffers a single point failure nor demands the use of a high-speed communication network. However, a low to medium bandwidth communication link is needed, which plays an important role in the overall optimization of the system operation. Most of the difficulties associated with decentralized and centralized control are overcome in this approach. The hierarchical control provides continuous operation at suboptimal level during a failure of the controller or communication network.

In the existing literature, various control schemes for DC MGs have been reported. Authors in [2] presented the drawbacks of conventional droop control and showed the effect of different droop gains on the current sharing accuracy and voltage regulation. The current sharing accuracy changes with the higher value of line impedance while reduction in voltage. Authors in [3] proposed and analyzed 3 nonlinear droop control techniques to obtain the optimal operation for load sharing accuracy and bus voltage restoration. A power distributed control method for proportional load power sharing and bus voltage restoration is reported in [4]. The proportional output power sharing with load voltage restoration is achieved by adjusting only the output source voltage instead of introducing the virtual impedance. However, poor load sharing at light load and voltage deviation in heavy load conditions persist in the system. Frequency droop control method is proposed in [5]. Fuzzy logic is used to define the frequency droop coefficient. Authors in [6] proposed and evaluated a fault detection method which is independent of the MG topology. Authors in [7] proposed a decentralized controller with low bandwidth communication to enhance high reliability, low voltage regulation, and equal load sharing. Eigenvalue analysis and mathematical modal were derived. The conventional droop curve equation is shifted along with $\mathrm{x}$-axis by adding additional voltage. A similar approach has been presented in [8], but the compensating average voltage of the identified critical busses is determined by the proposed modal analysis.

The hierarchical architecture was proposed for DC microgrid with reliable and optimum utilization of resources in $[9,10]$. Primary level control is embedded in Local Controllers (LCs) directly acting on power converters according to the references generated by the secondary level controller with faster dynamics. The secondary level control facilitates functions like bus voltage regulation, power management, and overall system optimization. Authors in [11] proposed a power management control strategy to DC MGs under different operating conditions. The developed control algorithm ensures optimal utilization of the available resources and maintains a healthy State of Charge (SoC) of the ESS. A two-level hierarchical control algorithm is proposed in [12] that can operate the main source at constant power and reduce the fluctuations on the voltage-controlled source in system while maintaining the SoC of the storage unit. Authors in [13] addressed the Adaptive Dynamic Power Management Strategy (ADPMS) for a self-reliant DC MG. Authors in [14] presented the design and implementation of a low voltage autonomous
MG integrating different RES combination with suitable energy storage units to meet the electrical energy needs in remote locations. It consists of a single voltage source converter and a Bidirectional DC Converter (BDDC) to manage the power flow in self-sustain low voltage MGs.

Emergency power and energy management system are essential in ensuring the optimal restoration process and continue the healthy operational amongst available RESs, energy storage system. Authors in [15] proposed a multi-level novel reconfigurable hierarchical architecture for an AC MG to transfer its functionalities from a source-level control to system level control to supports long-term optimization of $\mathrm{MG}$ operation under normal conditions and front-line switching roles in emergency conditions. Authors in [16] proposed the design and control of integrated RESs for energy management. The peak load demand problem was resolved with a combination of demand side energy management and ESSs. Authors in [17] proposed a methodology of making a schedule of list of actions such that the distribution system operator is able to provide continuous electricity supply for classified customers. This is achieved by considering some power management options such as intentional load shedding, dispatch of expensive fossil fuel sources, system reconfiguration, and so on. Authors in [18] presented a mathematical formulation based on mixed integer linear programming under normal and emergency operating conditions for an AC MG to improve the system resilience.

As discussed above, the focus of the research in DC MGs is more on power management under steady-state operation. Not much research is reported in operational management under emergency operating conditions. Therefore, it is essential to make more investigations in emergency cases to extend the MG operation in such emergency. This paper describes the novel Coordinated Power Management Strategy (CPMS) implemented on a typical LVDC MG architecture shown in Figure 2. The proposed CPMS facilitates LVDC MG operation under both steady-state and emergency conditions. The proposed strategy incorporates 3 levels of hierarchical control. The details of the proposed strategy are provided along with the simulation and the experimental results. The proposed strategy makes a LVDC MG a fault-resilient system able survive and perform under all operating conditions.

\section{THE COORDINATED POWER MANAGEMENT STRATEGY FOR THE LVDC MICROGRID}

An MG's main objective is to provide reliable power under all operating scenarios and this demands the use of a failsafe power management strategy. It is also essential to use the locally available resources optimally. The main focus is to maximize the power extraction from RES and to relieve the ESS from its major share in supplying power to the loads. With the abovementioned objectives, a 3 layered hierarchical control strategy is proposed for an autonomous LVDC MG. The power topology of the proposed LVDC MG considered for this study integrates 2 solar PV power plants and a fuel cell-based source as RES components while the ESS consists of battery-based and ultra-capacitor based sources. 


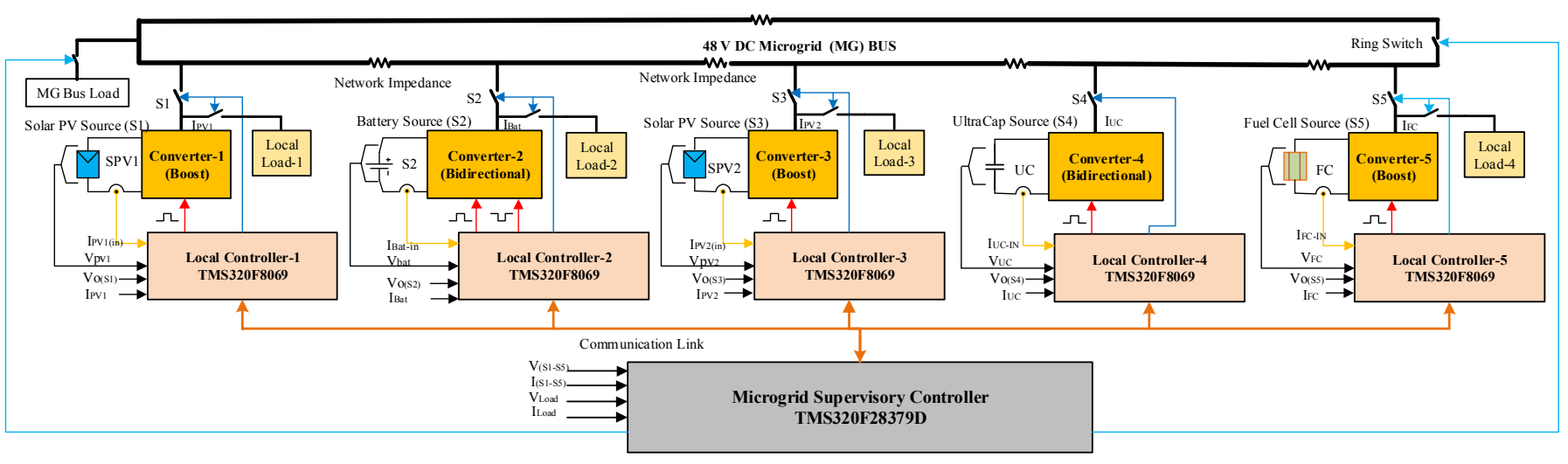

Fig. 2. MG power architecture of the proposed control strategy.

The battery-based source is meant for long term power backup and the ultracapacitor-based source is designed to meet the transient load requirements. Figure 2 shows the schematic of the complete LVDC MG. The boost converters with SPV are designed to operate in two modes: Maximum Power Point Tracking (MPPT) mode and Constant Current Mode (CCM). Similarly, a Bidirectional Converter (BDC) with battery and ultracapacitor is configured to operate in Voltage Control Mode (VCM) as well as in CCM when absorbing the excess power. The FC-based source with the boost converter is configured to work in both VCM and CCM.

\section{A. The Proposed Three Layer Hierarchical Control}

The hierarchical control is divided into 3 levels: local control, emergency control, and supervisory control as shown in Figure 3. The local control acts as the primary control directly on the DC-DC converters interfaced with each source. This includes source optimization and local power/energy management of each source. The top layer consists of supervisor control which optimizes the overall system performance while ensuring system stability. The emergency layer monitors the source and system parameters to ensure that their values are within the limits. In case of any parameter crossing the set limits, the emergency control layer intervenes with the local or supervisory control to initiate the necessary actions. In case of emergencies like a severe MG bus fault, the emergency control initiates the actions directly to ensure no damage occurs to the system components.

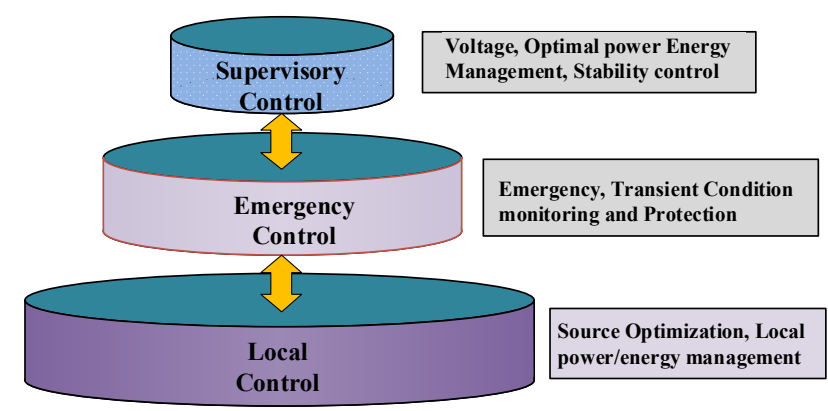

Fig. 3. The proposed three layer hierarchical control structure.
The converter of each source is designed with selfsustainable local control to work independently and feed the power to its own local loads. The supervisory control is responsible to control various primary controller parameters as and when required. The low band-width communication link between the supervisory and the local controller ensures the information exchange needed for the control and the coordination of power generation balancing and load demand. It also ensures the source and system level data for identifying the transient/emergency conditions for changing the mode of operations, as well as the calculation of the reference signals to source level primary controller parameters. The overall MG is designed to provide $3 \mathrm{~kW}$ of DC load at $48 \mathrm{~V}$ bus voltage. The specifications of the various power converter components and their ratings used in the hardware implementation are given in Table I.

TABLE I. POWER CONVERTER DETAILS OF THE STUDIED DC MG

\begin{tabular}{|c|c|c|c|c|c|c|c|}
\hline Converter & $\mathbf{V}_{\mathbf{S}}$ & $\begin{array}{c}\mathbf{P}_{\text {OUT }} \\
(\mathbf{k W})\end{array}$ & $\begin{array}{c}\mathbf{L} \\
(\mathbf{m H})\end{array}$ & $\begin{array}{c}\mathbf{E S R}_{\mathbf{L}} \\
(\mathbf{m} \mathbf{\Omega})\end{array}$ & $\begin{array}{c}\mathbf{C} \\
(\boldsymbol{\mu} \mathbf{F}) / \mathbf{V}\end{array}$ & $\begin{array}{c}\mathbf{E S R}_{\mathbf{C}} \\
(\mathbf{\Omega})\end{array}$ & $\begin{array}{c}\text { Conv. } \\
\text { Switch }\end{array}$ \\
\hline $\begin{array}{c}\text { Battery } \\
\text { BDC }\end{array}$ & $24 \mathrm{~V}$ & 1 & $\begin{array}{c}0.56 \\
50 \mathrm{~A}\end{array}$ & 10 & $\begin{array}{c}470 / \\
200 \mathrm{~V}\end{array}$ & 0.05 & $\begin{array}{c}\text { SKM100 } \\
\text { GB063D }\end{array}$ \\
\hline $\begin{array}{c}\text { SPV-boost } \\
\text { conv.1\&2 }\end{array}$ & $31 \mathrm{~V}$ & 1 & $\begin{array}{c}0.26 \\
50 \mathrm{~A}\end{array}$ & 6 & $\begin{array}{c}1000 / \\
200 \mathrm{~V}\end{array}$ & 0.1 & $\begin{array}{c}\text { SKM 100 } \\
\text { GB 063D }\end{array}$ \\
\hline Fuel cell & $24 \mathrm{~V}$ & 1.2 & $\begin{array}{c}0.56 \\
50 \mathrm{~A}\end{array}$ & 10 & $\begin{array}{l}1000 / \\
200 \mathrm{~V}\end{array}$ & 0.1 & $\begin{array}{c}\text { SKM 100 } \\
\text { GB 063D }\end{array}$ \\
\hline $\begin{array}{c}\text { Ultra- } \\
\text { capacitor } \\
\text { 165F/48V }\end{array}$ & $24 \mathrm{~V}$ & -- & $\begin{array}{c}0.26 \\
50 \mathrm{~A}\end{array}$ & 6 & $\begin{array}{c}470 / \\
200 \mathrm{~V}\end{array}$ & 0.05 & $\begin{array}{c}\text { SKM 100 } \\
\text { GB 063D }\end{array}$ \\
\hline
\end{tabular}

The switching frequency for all the converters is selected to be $20 \mathrm{kHz}$. The supervisory control is realized on a Texas Instrument's TMS320F28379D dual core controller board whereas the local control for each source is realized on TMS320F28069 controller boards. Based on hierarchical control strategy, the supervisory controller selects the mode of operation for each local converter through serial communication executed on the DSP. Figure 4 shows the power and control scheme implemented for various converters. The details of design of both VCM and CCM are given in [10]. The emulated fuel cell source is designed to be similar to a battery based source except the unidirectional boost converter instead of the BDC. Figure 5 shows a photograph of the 
developed LVDC MG in hardware with the specifications listed in Table I. The functionalities of the proposed control strategy were verified with simulations carried out in the PSIM platform. The simulations were carried out to investigate some technical operational challenges and the way they were addressed suitably. Various operating scenarios under normal and emergency conditions were created as cases listed below. Also, hardware implementation of power topology along with the proposed control scheme was carried out to validate the results.

(a)
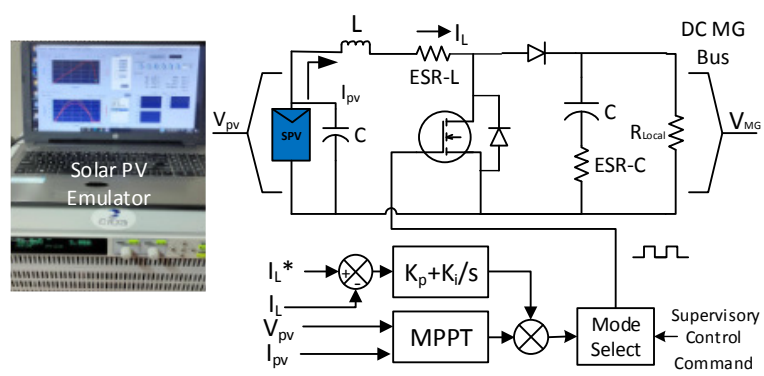

(b)
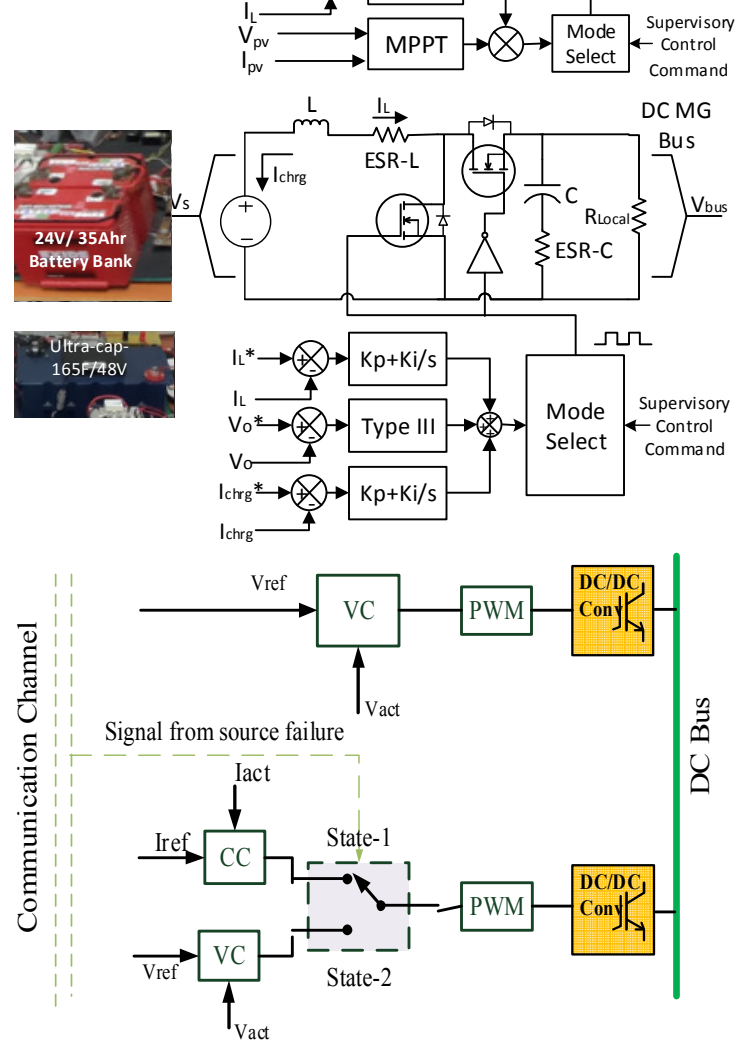

Fig. 4. (a) Solar PV 1 and 2 boost converter with CCM and MPPT control mode. (b) Battery and ultra-capacitor boost converters with VCM and CCM control mode. (c) Mode select control logic with communication received from the supervisory controller.

\section{B. CPMS Simulation Results in Normal Operating Conditions}

The hierarchical control based algorithm ensures the continuity of supply and efficient source utilization under all operating conditions. The supervisory control mentors the local variable parameters from the local controllers to perform the system level operating modes and passes the charging/discharging information. Considering the different power generation and loading scenarios for the proposed DC microgrid for urban applications, a few Normal Operational Scenarios (NOS) are describing as below.

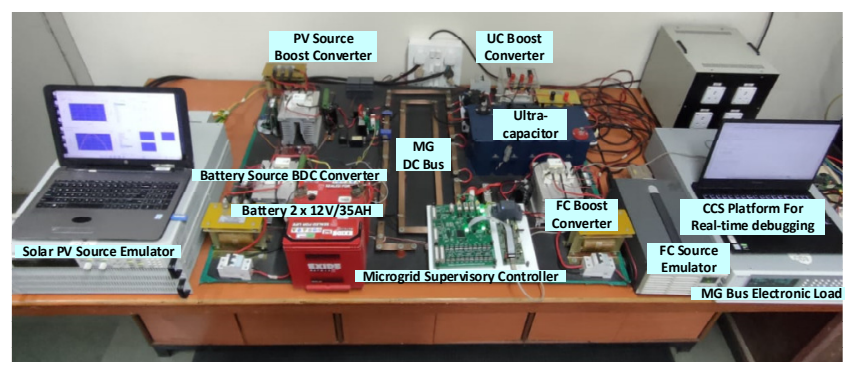

Fig. 5. Photograph of the developed LVDC MG setup.

NOS-1: During the light load conditions, the MG bus load is fed from the SPV and battery sources. As seen in Figure 6, the SPV source 1 is operating in MPPT mode to supply the primarily load current. The battery source maintains the MG bus voltage by absorbing the excess power or delivering the deficit power. The load is reduced at $t=2 \mathrm{~s}$, the excess power from the SPV 1 is absorbed by the battery. Before $t=2 \mathrm{~s}$, the load demand is met by SPV 1 and the battery.

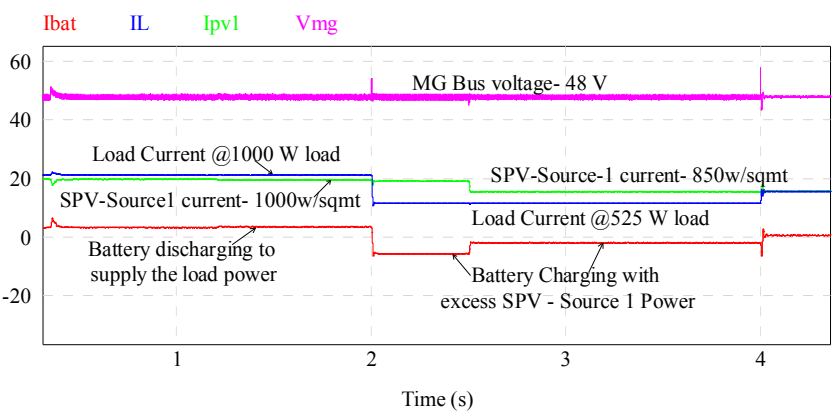

Fig. 6. NOS 1: Light load condition supported with SPV and battery.

NOS-2: Under light load conditions, load is fed by the SPV and battery sources, but if the SPV power is more than the connected MG bus load, then battery charging is carried out. Figure 7 shows, the solar PV power generation changes as per solar insolation level. The SPV source supplies the available power to the load in the MPPT mode.

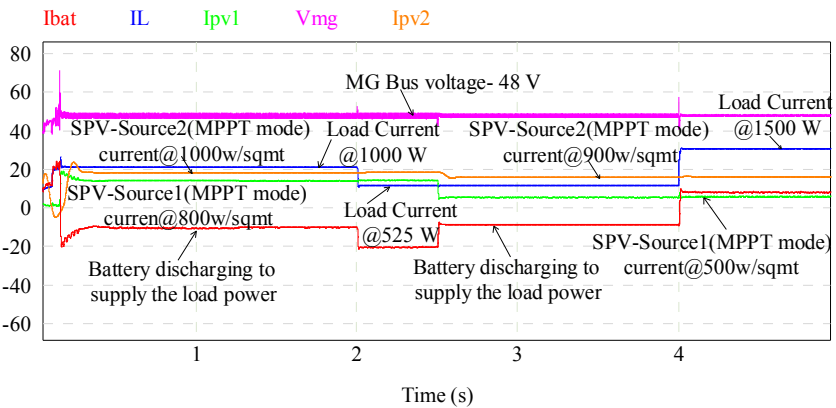

Fig. 7. NOS 2: Light load condition supported with SPV and battery with opportunity charging scenario. 
The battery source maintains the MG bus voltage by absorbing the excess power or delivering the deficit power. The battery can be availed with opportunity charging in case the PV power is more than the instantaneous load demand. At $t=2.5 \mathrm{~s}$ the solar insulation level is changed and excess power from solar PV 1 and 2 is utilized to battery charging.

NOS-3: The MG bus load increased from $0.5 \mathrm{~kW}$ to $2.8 \mathrm{~kW}$. The SPV sources 1 and 2 are both operating in MPPT mode primarily delivering the available power to the load. Fuel cellbased source working in current controlled mode supplements the SPV sources feeding the load. The battery source maintains the MG bus voltage by absorbing the excess power or delivering the deficit power. The simulation results for full load condition with the fuel cell operating in CC mode supplemented by both SPV sources is shown in Figure 8. Under full load conditions, all the sources participate to feed the MFG bus load while both the SPV sources deliver the available maximum power.

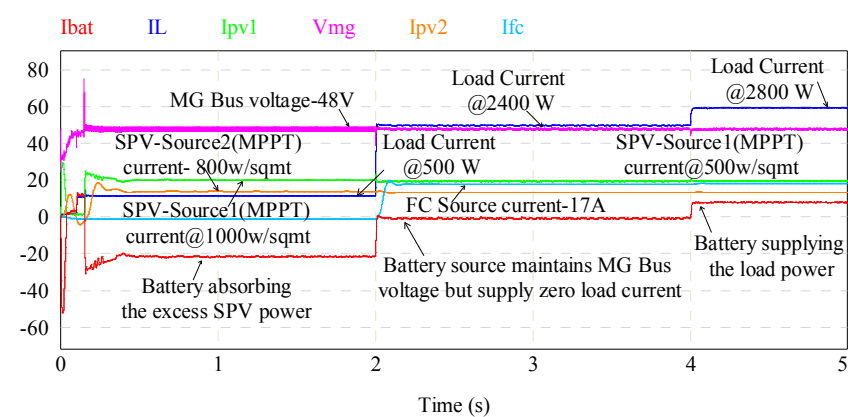

Fig. 8. NOS 3:-Full load condition supported with SPV (MPPT mode), fuel cell (CC mode), and battery sources (CV mode).

NOS-4: The MG bus load increased from $0.5 \mathrm{~kW}$ to $2.8 \mathrm{~kW}$. SPV sources 1 and 2 are both operating in CC mode. The fuel cell source is configured to deliver the available power to the load while maintaining the MG bus voltage. The battery source delivers no load power but is engaged in charging through the $\mathrm{FC}$ as can be seen in Figure 9.

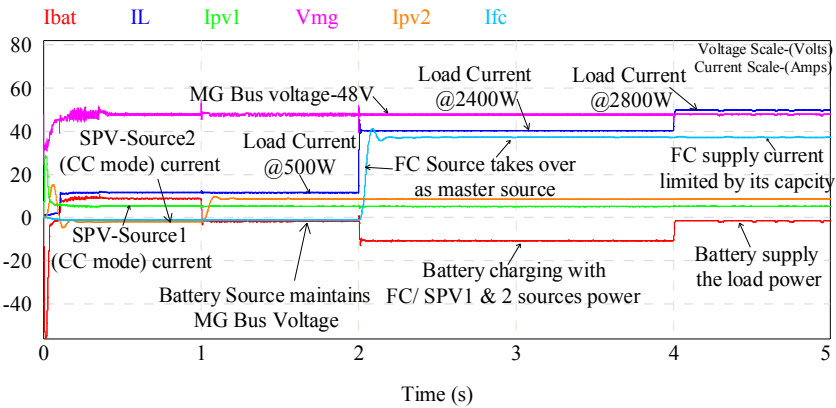

Fig. 9. NOS 4: Full load condition supported with SPV (CC mode), fuel cell (CV mode), and battery sources (charging mode).

NOS-5: The SPV sources 1 and 2 are both operating in CC mode. The battery source is configured to primary deliver the available power to the load while maintaining the MG bus voltage. At $1.5 \mathrm{~s}$, the capacitive load imposed on the MG bus results in large transient current requirement. The ultracapacitor source pumps the demanded transient current, thus relives the battery from supplying the transient load. The transient phase mitigation by the ultracapacitor source is shown in Figure 10.

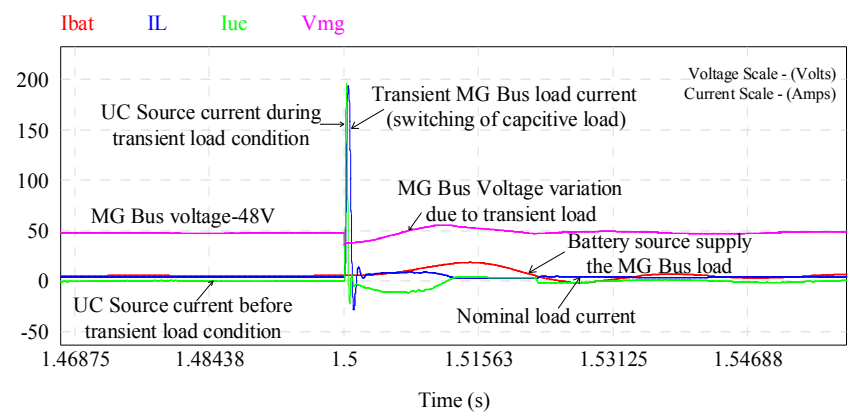

Fig. 10. NOS 5: Transient loading condition.

\section{CPMS Simulation Results in Emergency Operating Conditions}

Emergency conditions such as MG short bus faults, failure of controllers/sources, loss of generation, and heavy overloads can occur in a DC MG. However, in the extreme case of a fault, the PMS may not be capable of retaining the MG in normal operating condition. When it comes to improving the resiliency and the reliability of the DC MG, power management and coordination strategy play a vital role. It is assumed that the fault was occurred, detected, and isolated by the emergency control layer and then control and coordination actions have to react quickly ensuring stability. Considering the different power generation and loading scenarios for the proposed DC MG, a few emergency operating scenarios (EOSs) are described below.

EOS-1: The SPV sources 1 and 2 are both operating in MPPT mode. The battery source is configured to primarily deliver the available power to the load while maintaining the MG bus voltage. At $2.5 \mathrm{~s}$, due to passing clouds, the power available from the SPV sources to zero. The battery source pumps additional load current to maintain the MG bus voltage. Figure 11 shows the simulation results of a sudden loss of the non-dispatchable SPV source.

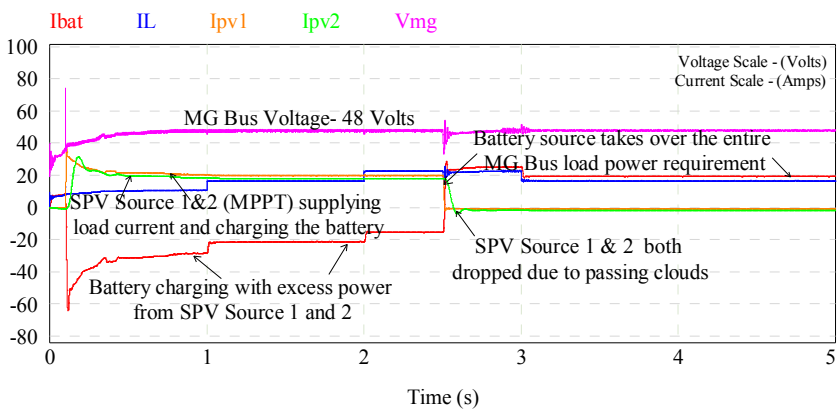

Fig. 11. EOS 1: Sudden loss of the non-dispatchable SPV source. 
EOS-2: The SPV sources 1 and 2 are both operating in MPPT mode. The battery is assumed to be charging with the excess power from the SPV sources. At $1.5 \mathrm{~s}$, the SPV Source 2 suffers from loss of generation. At $2 \mathrm{~s}$, due to the increase in load demands, the fuel cell source kicks in to supply the additional load current to maintain the MG bus voltage. The simulation results are shown in Figure 12.

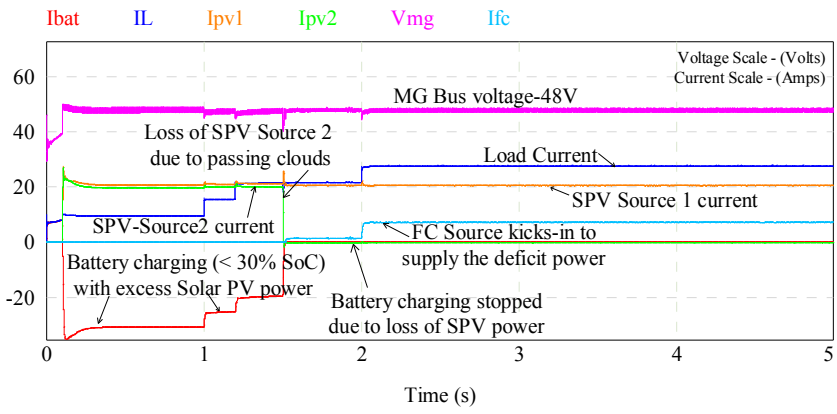

Fig. 12. EOS 2: Sudden loss of the dispatchable battery source.

\section{HARDWARE RESULTS}

A laboratory scaled prototype (Figure 5) was designed and implemented for validation of the proposed $\mathrm{MG}$ with hierarchical control to supply $3 \mathrm{~kW}$ DC load at $48 \mathrm{~V}$. Some selected hardware results are presented in Figures 13-16 indicating the various parametric measurements and their dynamic responses under different operating conditions (only few illustrative results are shown in this paper). Figure 13 shows the SPV source and battery source managing the overall load requirement of the MG under light load conditions. Figure 13(a) shows the available SPV power changes as per the variation in the solar insolation and the deficit power that is then delivered by the battery source ensuring the MG bus voltage is maintained within the specified allowable limit. Figure 13(b) shows the SPV source emulation with the MPPT mode primarily delivering the available power to the load while maintaining MG bus at $48 \mathrm{~V}$ throughout the operation.

Figure 14 shows the emulated fuel cell and battery sources managing the overall load requirements of the MG. The SPV source is operating in MPPT mode primarily delivering the available power to the load. This situation may arise when the SPV source is not available, e.g. under rainy conditions or during the night. The fuel cell-based source working in current controlled mode supplements the battery source feeding the load. The battery source maintains the MG bus voltage by delivering the deficit power. In normal operating conditions, it is ensured that the SPV and the battery handle the load requirement. Under heavy load conditions, the fuel cell source along with the SPV and battery sources are utilized to supply the net MG bus load, whereas in light load conditions, the SPV source operating in MPPT mode delivers the excess power to the battery. Figure 15 shows the opportunity charging of the battery source when the SPV source power at the MPPT is more than the one demanded by the MG bus load. The battery source gets charged through the excess SPV source power while maintaining the MG bus voltage.

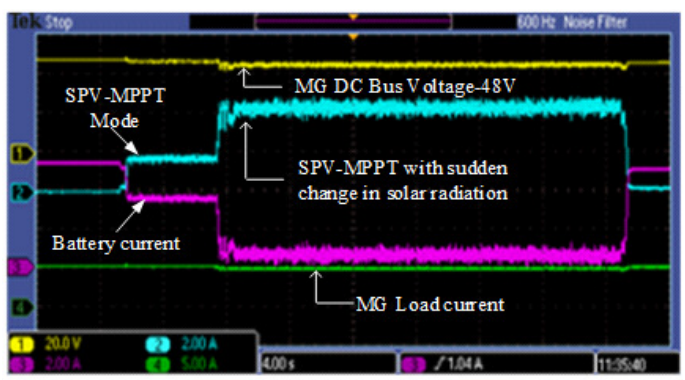

(a)

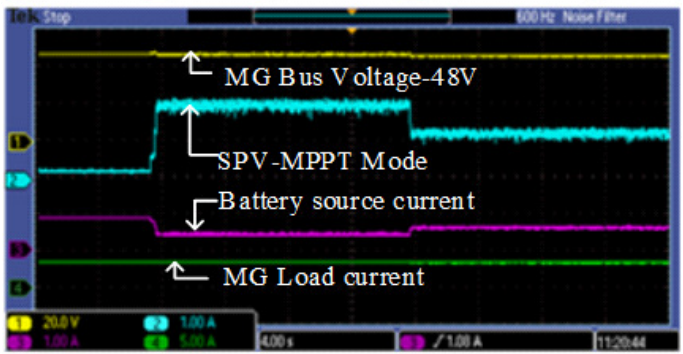

(b)

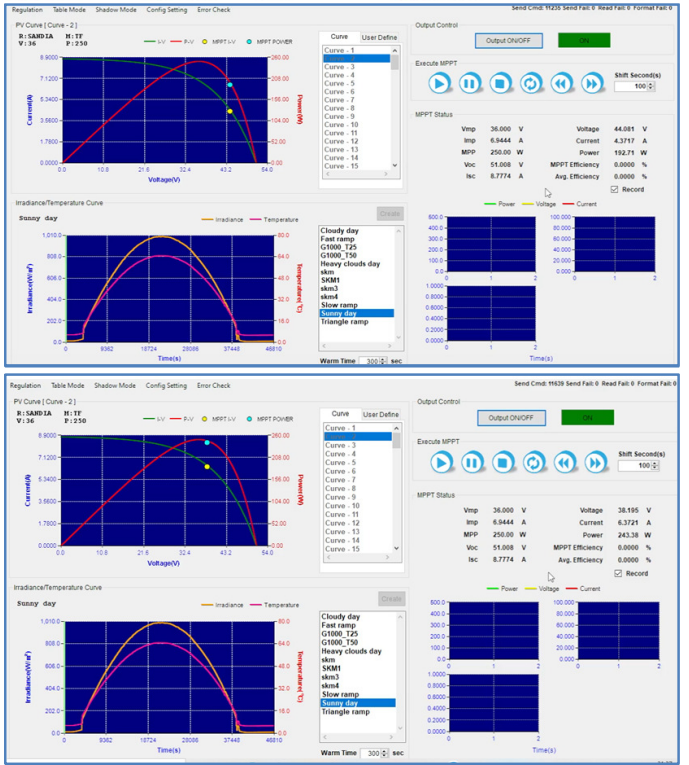

Fig. 13. (a) The SPV source is in the MPPT mode primarily to supply the load current and the battery source maintains the MG bus voltage by absorbing the excess power or delivering the deficit powerage due to solar insolation changes. (b) SPV emulation with MPPT tracking.

Under transient loads, like switching of capacitive loads, or short term fault conditions, the current drained from the battery source can be very high which may lead to the faster deterioration of the battery cells thus reducing the life of the battery. As shown in Figure 16, under such conditions the UC source gets engaged due to the faster current loop design and hence the faster dynamic response.

Under any transient current demand conditions, the UC source supplies the current, thus reducing the current requirement demanded from the battery source. The hardware setup of the LVDC MG with the proposed coordinated power management strategy implemented was tested under various other conditions as explained in the simulation results. 
(a)

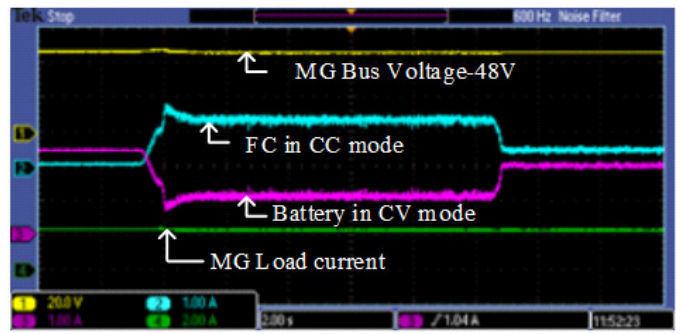

(b)

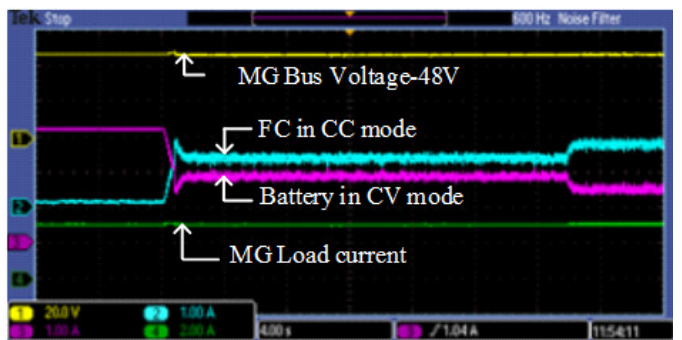

Fig. 14. (a) FC (CC mode) and battery ( $\mathrm{CV}$ mode) under a particular loading condition. (b) The FC source feeds designated power to the load based on the command received from the CPM, and the battery source supply the deficit power.

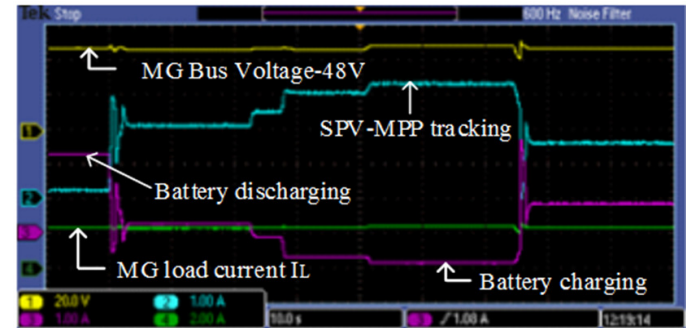

Fig. 15. SPV source with MPPT mode delivering available power to load and excess power under light load condition is supplied to the battery source with opportunity charging.

(a)

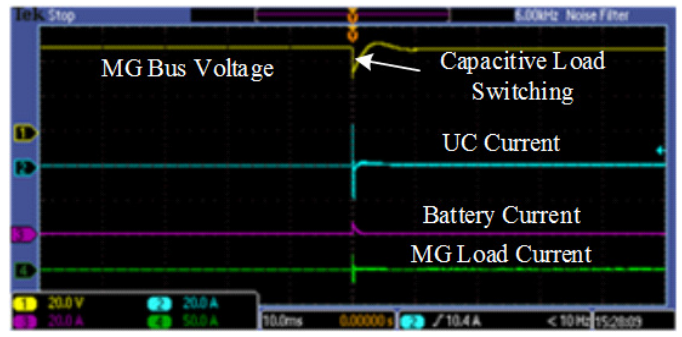

(b)

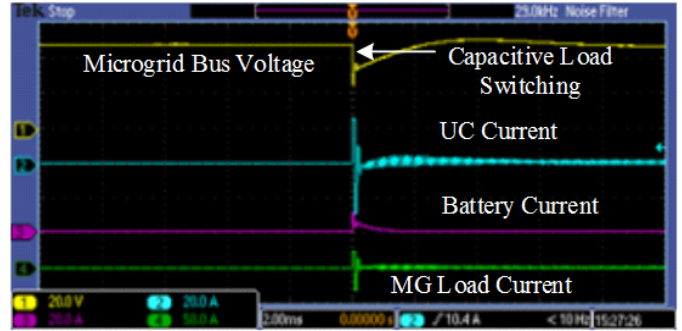

Fig. 16. (a) Capacitive load switching: The UC source contributes the transient current relieving the battery source. (b) Expanded view of (a).
The performance of the MG proved to satisfy the requirement of reliable MG bus supply under all the operating conditions. Because a DC MG involves the integration of different types of renewable energy sources with dynamical changing characteristics and continuous changing in loads, $\mathrm{MG}$ control needs to be designed to ensure that the bus voltage stability is maintained all the time. The power sharing between the MG sources which ensures flat MG bus voltage profile under any operational conditions while reliably fulfilling the load requirements is a challenging task. Most of the researchers focused on power sharing under normal operating conditions while at emergency conditions the focus was on the protection of the system. While some researchers have already addressed the suitable power management strategies like adaptive dynamic power management strategy, different combinations of RESs with suitable energy storage units, demand side energy management and ESSs, intentional load shedding, dispatch of expensive fossil fuel sources, system reconfiguration by considering one by one the issues to be taken into the account under normal operating conditions. Very few papers have reported a power sharing strategy under emergency operating conditions in DC MGs. Under emergency conditions the demand in control and coordination between various subsystems of the MG to survive and extend the MG operation may significant change. This paper presented a highly effective hierarchical control with coordinated power management strategy for MG sources and ESSs to handle both normal and abnormal situations. The proposed control strategy was validated with simulation and experimental results in an implemented LVDC MG to showcase its reliable behavior under normal and emergency conditions.

\section{CONCLUSION}

This paper presented the implementation of a hierarchically controlled LVDC MG with SPV, fuel cell source as RES components, and ESS comprised of battery based and ultracapacitor based sources. The proposed LVDC MG system can facilitate the integration of such RESs to supply continuous power to the load. The proposed coordinated power management strategy based on hierarchical control for an autonomous LVDC MG ensures the reliable operation of the system with renewable energy sources, storage, and loads. The proposed strategy ensures the proper power sharing and the continuation of the MG operation under normal and emergency conditions. The hierarchically controlled DC MG operating in autonomous mode under normal and emergency conditions has been tested and some of the hardware results were reported.

\section{REFERENCES}

[1] X. Lu, K. Sun, J. M. Guerrero, J. C. Vasquez, and L. Huang, "State-ofCharge Balance Using Adaptive Droop Control for Distributed Energy Storage Systems in DC Microgrid Applications," IEEE Transactions on Industrial Electronics, vol. 61, no. 6, pp. 2804-2815, Jun. 2014, https://doi.org/10.1109/TIE.2013.2279374.

[2] A. Khorsandi, M. Ashourloo, and H. Mokhtari, "A Decentralized Control Method for a Low-Voltage DC Microgrid," IEEE Transactions on Energy Conversion, vol. 29, no. 4, pp. 793-801, Dec. 2014 https://doi.org/10.1109/TEC.2014.2329236.

[3] P. Prabhakaran, Y. Goyal, and V. Agarwal, "Novel Nonlinear Droop Control Techniques to Overcome the Load Sharing and Voltage Regulation Issues in DC Microgrid," IEEE Transactions on Power 
Electronics, vol. 33, no. 5, pp. 4477-4487, May 2018, https://doi.org/ 10.1109/TPEL.2017.2723045.

[4] D.-H. Dam and H.-H. Lee, "A Power Distributed Control Method for Proportional Load Power Sharing and Bus Voltage Restoration in a DC Microgrid," IEEE Transactions on Industry Applications, vol. 54, no. 4, pp. 3616-3625, Jul. 2018, https://doi.org/10.1109/TIA.2018.2815661.

[5] P. D. Chung, "Retaining of Frequency in Micro-grid with Wind Turbine and Diesel Generator," Engineering, Technology \& Applied Science Research, vol. 8, no. 6, pp. 3646-3651, Dec. 2018, https://doi.org/ 10.48084/etasr.2413

[6] R. Eslami, S. H. H. Sadeghi, and H. A. Abyaneh, "A Probabilistic Approach for the Evaluation of Fault Detection Schemes in Microgrids," Engineering, Technology \& Applied Science Research, vol. 7, no. 5, pp. 1967-1973, Oct. 2017, https://doi.org/10.48084/etasr.1472.

[7] S. Anand, B. G. Fernandes, and J. Guerrero, "Distributed Control to Ensure Proportional Load Sharing and Improve Voltage Regulation in Low-Voltage DC Microgrids," IEEE Transactions on Power Electronics, vol. 28, no. 4, pp. 1900-1913, Apr. 2013, https://doi.org/ 10.1109/TPEL.2012.2215055.

[8] S. Peyghami, H. Mokhtari, P. Davari, P. C. Loh, and F. Blaabjerg, "On Secondary Control Approaches for Voltage Regulation in DC Microgrids," IEEE Transactions on Industry Applications, vol. 53, no. 5, pp. 4855-4862, Sep. 2017, https://doi.org/10.1109/TIA.2017.2704908.

[9] T. Dragičević, J. M. Guerrero, J. C. Vasquez, and D. Škrlec, "Supervisory Control of an Adaptive-Droop Regulated DC Microgrid With Battery Management Capability," IEEE Transactions on Power Electronics, vol. 29, no. 2, pp. 695-706, Feb. 2014, https://doi.org/ 10.1109/TPEL.2013.2257857.

[10] S. Muchande and S. Thale, "Design and Implementation of Autonomous Low Voltage DC Microgrid with Hierarchical Control," in 2020 IEEE First International Conference on Smart Technologies for Power, Energy and Control (STPEC), Nagpur, India, Sep. 2020, https://doi.org/10.1109/STPEC49749.2020.9297748.

[11] R. Pradhan, M. Chirayath, and S. Thale, "Coordinated control strategy for a DC microgrid with low bandwidth communication," in 2016 IEEE International Conference on Power Electronics, Drives and Energy Systems (PEDES), Trivandrum, India, Dec. 2016, https://doi.org/ 10.1109/PEDES.2016.7914276.

[12] R. R. Deshmukh, M. S. Ballal, H. M. Suryawanshi, and G. G. Talapur, "A control algorithm for energy management and transient mitigation in DC microgrid," in 2017 National Power Electronics Conference (NPEC), Pune, India, Dec. 2017, pp. 270-275, https://doi.org/ 10.1109/NPEC.2017.8310470.

[13] B. R. Naidu, G. Panda, and P. Siano, "A Self-Reliant DC Microgrid: Sizing, Control, Adaptive Dynamic Power Management, and Experimental Analysis," IEEE Transactions on Industrial Informatics, vol. 14, no. 8, pp. 3300-3313, Aug. 2018, https://doi.org/10. 1109/TII.2017.2780193.

[14] Seema and B. Singh, "PV-Hydro-Battery Based Standalone Microgrid for Rural Electrification," in 2018 5th IEEE Uttar Pradesh Section International Conference on Electrical, Electronics and Computer Engineering (UPCON), Gorakhpur, India, Nov. 2018, https://doi.org/ 10.1109/UPCON.2018.8597005.

[15] S. S. Thale, R. G. Wandhare, and V. Agarwal, "A Novel Reconfigurable Microgrid Architecture With Renewable Energy Sources and Storage," IEEE Transactions on Industry Applications, vol. 51, no. 2, pp. 18051816, Mar. 2015, https://doi.org/10.1109/TIA.2014.2350083.

[16] T. V. Krishna, M. K. Maharana, and C. K. Panigrahi, "Integrated Design and Control of Renewable Energy Sources for Energy Management," Engineering, Technology \& Applied Science Research, vol. 10, no. 3, pp. 5857-5863, Jun. 2020, https://doi.org/10.48084/etasr.3613.

[17] D. Q. Oliveira, A. C. Zambroni de Souza, A. B. Almeida, M. V. Santos, B. I. L. Lopes, and D. Marujo, "Microgrid management in emergency scenarios for smart electrical energy usage," in 2015 IEEE Eindhoven PowerTech, Eindhoven, Netherlands, Jun. 2015, https://doi.org/10. 1109/PTC.2015.7232309.

[18] M. AlOwaifeer, A. Alamri, and A. P. S. Meliopoulos, "Microgrid Energy Management System for Normal and Emergency Operating
Conditions," in 2019 North American Power Symposium (NAPS), Oct. 2019, https://doi.org/10.1109/NAPS46351.2019.9000218.

\section{AUTHORS PROFILE}

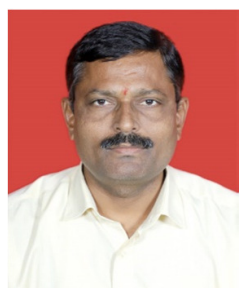

Shrishell Muchande completed his B.E. and M.E degrees in Electrical Engineering at the University of Mumbai, Mumbai, India, in 2008 and 2013, respectively. Since 2016 , he is a research scholar in the Department of Electrical Engineering, Fr. Conceicao Rodrigues Institute of Technology, Navi Mumbai, Mumbai, India. He has over 4 years of experience in industry. His interest areas include power electronics, renewable energy systems, microgrids, and control in microgrids. From 1996 to 2012, he was worked at the Lokmanya Tilak Collage of Engineering, Koparkhairane, Navi Mumbai. Since 2013, he has been a member of the Faculty of the Department of Electronics and Telecommunication Engineering, Gharda Institute of Technology, Lavel, Ratnagiri, Maharastra, India.

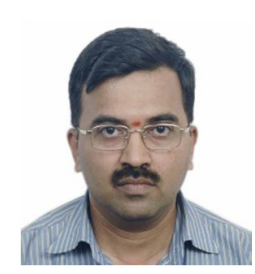

Sushil Thale obtained his B.E. degree in Electrical Engineering in 1992 and M.E. in Electrical Engineering in 1996 from the University of Mumbai, India. He completed his Ph.D. at the Indian Institute of Technology-Bombay with specialization in Power Electronics and Power Systems in 2015. He is currently working as a Professor (Electrical) and Dean (R\&D) at Fr. Conceicao Rodrigues Institute of Technology, Navi Mumbai, India. His areas of research include renewable energy Systems, electric vehicle control, and microgrids. 